\title{
Potential sputtering of ionic species from rare gas solids by multiply charged ion impact
}

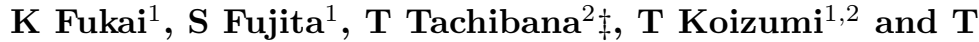 \\ Hirayama $^{1,2}$ \\ ${ }^{1}$ Department of Physics, Rikkyo University, Nishi-Ikebukuro, Toshima-ku, \\ Tokyo 171-8501, JAPAN \\ ${ }^{2}$ Research Center for Measurements in Advanced Science (RCMAS), Rikkyo \\ University, Nishi-Ikebukuro, Toshima-ku, Tokyo 171-8501, JAPAN \\ E-mail: hirayama@rikkyo.ac.jp
}

\begin{abstract}
The interaction of low-energy multiply charged $\mathrm{Ar}^{q+}(q \leq 7)$ ions with a solid Ne surface is experimentally studied. Desorption of very large cluster ions $\mathrm{Ne}_{n}^{+}(n>100)$ is observed. The size distribution of smaller $(n=1 \sim 3)$ cluster ions depends strongly on the charge state of the incident ion, whereas that of larger $(n>7)$ cluster ions exhibits no dependence on the charge state, indicating that desorption of large cluster ions is due to kinetic sputtering. The potential sputtering yield is estimated by analyzing the size distribution of the desorbed cluster ions. The results suggest that the ion desorption mechanism, which is known as desorption induced by electronic transitions, can also be applied to the present results.
\end{abstract}

\section{Introduction}

Rare-gas solids (RGS) are interesting materials for investigating the dynamic processes of desorption induced by electronic transitions (DIET) because of their simplicity and because their electronic structures resemble those of isolated atoms. Furthermore, they have several characteristics that differ greatly from those of other materials, including low cohesive energies (e.g., $0.02 \mathrm{eV} /$ atom for $\mathrm{Ne}$ ), large band-gap energies (e.g., 21.6 $\mathrm{eV}$ for $\mathrm{Ne}$ ), and long exciton diffusion lengths (e.g., $\sim 200 \mathrm{~nm}$ for Ne), etc. [1]

Desorption of atoms and ions by low-energy electron impact, known as electronstimulated desorption (ESD), or by VUV photon irradiation, known as photonstimulated desorption (PSD), from the surfaces of RGS has been extensively studied over the past two decades [2-4]. The creation of excitons and ions plays an important role in the desorption of neutral atoms, whereas the formation of an electronically excited ion or a doubly charged ion is essential for the desorption of singly charged ions [5]. This is also true for the desorption of doubly charged ions, where a coreexcited ion or a triply charged ion can be the initial state that leads to desorption [6].

Sputtering from RGS by singly charged $\mathrm{keV}$ ion impact has been investigated by various groups [7-13], and a kinetically induced sputtering mechanism has been discussed in detail.

$\ddagger$ Present address: Department of Physics, Tokyo University of Science, 1-3 Kagurazaka, Shinjukuku, Tokyo 162-8601, JAPAN 
Here, we report for the first time the potential sputtering of ionic species from the surface of solid Ne by low-energy multiply charged Ar ion impact, and we report the potential sputtering yield.

\section{Experimental}

Multiply charged ions $\left(\mathrm{Ar}^{q+}, q \leq 7\right)$ are generated by an electron cyclotron resonance ion source (NANOGAN). Extracted ions are mass-selected by an analyzing magnet and are focused onto the sample surface at the center of a main chamber (see Fig. 1). The main chamber is evacuated by a series of turbomolecular pumps and a Ti-getter pump, resulting in a pressure of about $8 \times 10^{-9} \mathrm{~Pa}$. Rare-gas solids are condensed onto a polycrystalline $\mathrm{Cu}$ substrate cooled to $4.5 \mathrm{~K}$ by a mechanical cryostat. The sample film is condensed onto the $\mathrm{Cu}$ disk by filling the chamber with gaseous $\mathrm{Ne}$ to a pressure of $10^{-6} \sim 10^{-4} \mathrm{~Pa}$. The film thickness was estimated from the exposure by assuming the condensation coefficient to be unity.

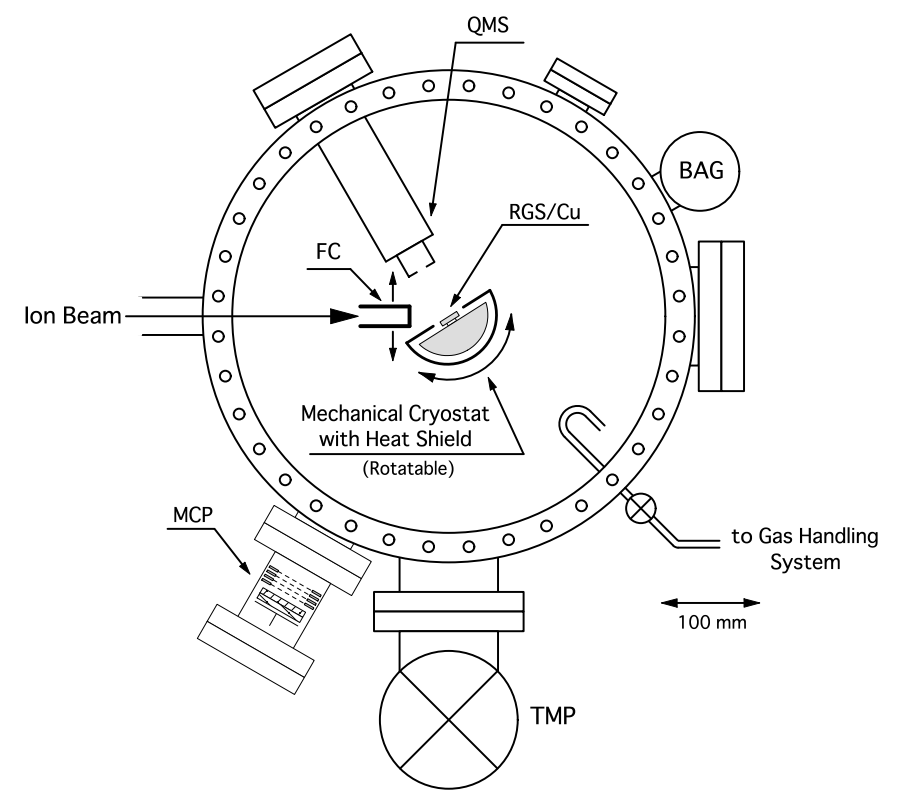

Figure 1. Schematic of the main chamber (top view). BAG: Bayard-Alpert ionization gauge, QMS: quadrupole mass spectrometer, MCP: microchannel plate detector, TMP: tandem turbomolecular pump, FC: movable Faraday cup.

Sputtered ionic species are detected by a microchannel plate (MCP) detector or a quadrupole mass spectrometer (QMS; MSQ-400, ULVAC) in pulse-counting mode. Time-of-flight (TOF) mass spectra are measured using the MCP detector by pulsing the incident ion beam, while a DC beam is used for obtaining mass spectra using the QMS. In the TOF measurements, a bias voltage of $+100 \mathrm{~V}$ is applied to the sample substrate to accelerate the sputtered ions. When using the QMS, the sample is grounded and the ionizer of the QMS is turned off. The incident ion beam current can be measured to a higher accuracy using the QMS than using a pulsed ion beam 
with the MCP detector, although the measurement range is restricted to $M / q \leq 400$ with the QMS used in this study.

As the desorption yield is very large for solid Ne [14], a low incident ion beam current (less than $1 \mathrm{nA}$ ) is used to minimize the change in sample thickness during measurement. The sample is evaporated and redeposited after each measurement.

\section{Results and Discussion}

Figure 2 shows TOF spectra of ions desorbed by $2.7 \mathrm{keV} \mathrm{Ar}{ }^{3+}$ ion impact. The figure shows that the main desorbing species are the cluster ions $\mathrm{Ne}_{n}^{+}$( $n$ : cluster size) and that very large clusters $(n>100)$ are present (Fig. 2(b)). Clear steps are observed (i.e., magic numbers at $n=14$ and 21), as also reported in supersonic cluster beam experiments [15]. The magic number $n=14$ is attributed to an icosahedral structure with a dimer ion at its core [16]. Similar spectra have been observed for low-energy $\mathrm{Ne}^{q+}$ impact but not for excitation by $100 \mathrm{eV}$ electron impact $\left(n_{\max } \sim 5\right)$. Desorption of such large cluster ions is not detected from the surface of solid $\operatorname{Ar}\left(n_{\max } \sim 20\right)$. A detailed discussion of the growth and desorption mechanism for such large cluster ions will be published elsewhere [17].
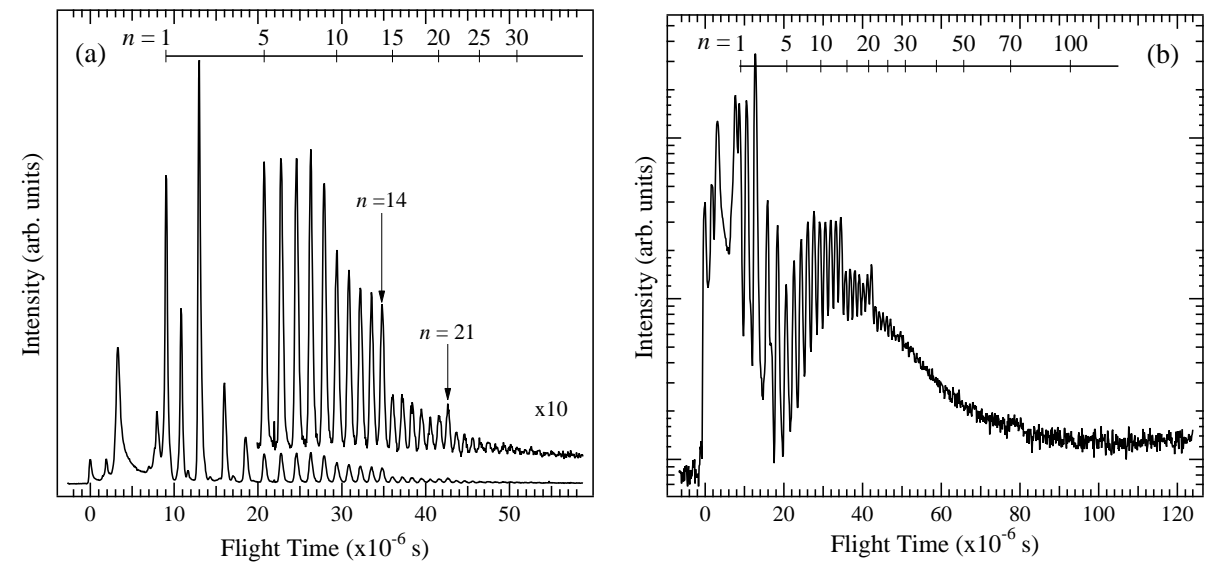

Figure 2. Time-of-flight spectra of desorbed ions from the surface of solid Ne by $2.7 \mathrm{keV} \mathrm{Ar}^{3+}$ ion impact plotted with (a) linear and (b) logarithmic vertical scales. The sample is about $600 \mathrm{ML}$ (mono-layers) thick. The size $n$ of the desorbed cluster ions $\mathrm{Ne}_{n}^{+}$is indicated in the figure.

Figure 3 shows the mass spectra of desorbed cluster ions from solid Ne by $1 \mathrm{keV}$ $\operatorname{Ar}^{q+}(q=1,3$, and 6$)$ ion impact measured by QMS. The vertical axis is the number of detected ions normalized by the number of incident ions (i.e., $I_{i} / q$ ). It clearly shows that the intensities of the smaller cluster ions $(n=1 \sim 3)$ depend strongly on the charge state of the incident ions. The peak area for each cluster size is plotted in Fig. 4 for $q=1,3,4,6$, and 7 , clearly demonstrating the charge state dependence of the size distribution. The figure shows that the desorption yield of larger clusters $(n>$ 7) does not depend on the charge state, indicating that the desorption mechanism of large cluster ions is kinetic sputtering.

Considering that the potential energy of $\mathrm{Ar}^{+}(15.76 \mathrm{eV})$ is less than the creation energies of an exciton $(17.1 \mathrm{eV})$ and an ion $(21.6 \mathrm{eV})$ in solid $\mathrm{Ne}[1]$, we assume that 


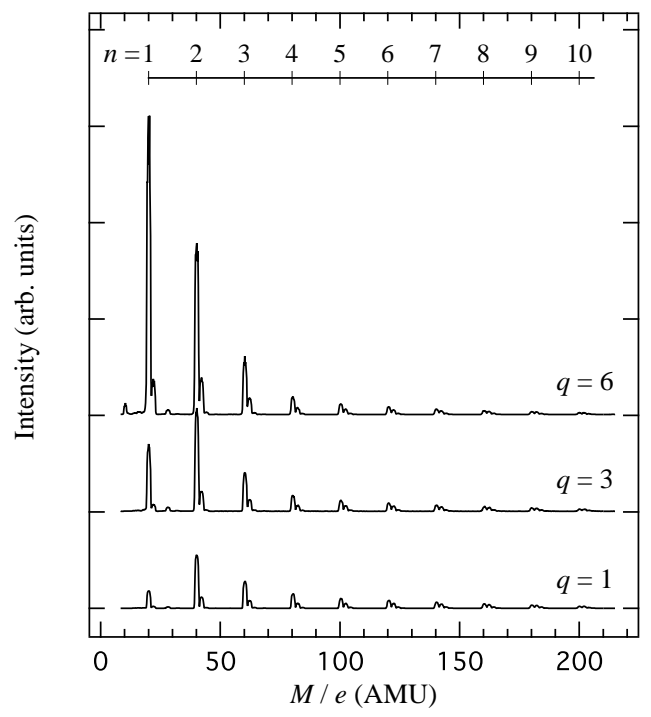

Figure 3. Mass spectra of desorbed cluster ions by $1 \mathrm{keV} \mathrm{Ar}^{q+}(q=1,3$, and 6 ) ion impact measured by the QMS. The vertical axis is the number of detected ions normalized by the number of incident ions.

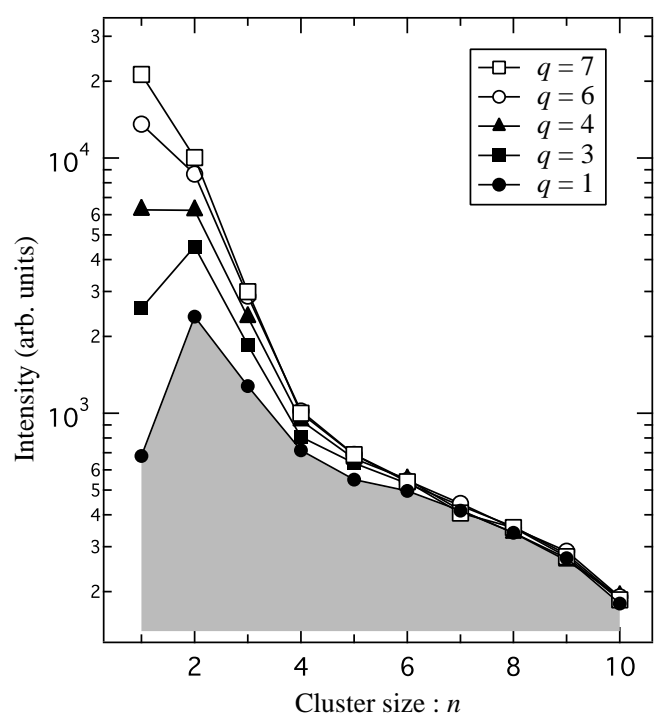

Figure 4. Dependence of the size distribution on the charge state of the incident ions. Incident energy of the ion is $1 \mathrm{keV}$ for all charge states. See text for details. 


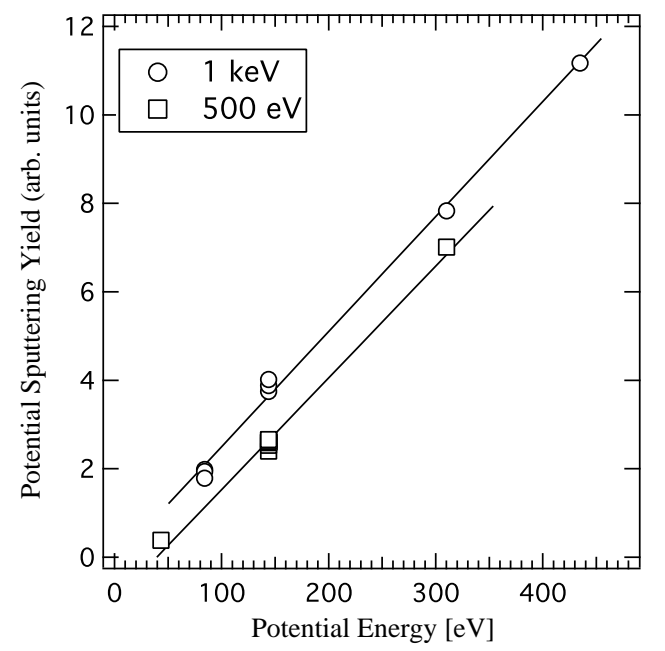

Figure 5. Potential sputtering yield of ionic species from the surface of solid $\mathrm{Ne}$ by $500 \mathrm{eV}(\square)$ and $1 \mathrm{keV}(\mathrm{O}) \mathrm{Ar}^{q+}(q=2,3,4,6,7)$ ion impact. The sample is 600 ML thick.

only kinetic sputtering occurs in the desorption of ionic species by $\mathrm{Ar}^{+}$ion impact. To estimate the potential sputtering yield (PSY), the contribution of kinetic sputtering (i.e., the sum of the peak areas of $\mathrm{Ar}^{+}$impact; shaded area in Fig. 4) is subtracted from the size distribution of $q \geq 2$. The results are plotted in Fig. 5 for incident ion energies $E_{i}$ of $500 \mathrm{eV}$ and $1 \mathrm{keV}$. The vertical axis in Fig. 4 corresponds to the number of charges (not atoms) per incident ion in arbitrary units. This figure shows that the slopes the incident energies of $E_{i}=500 \mathrm{eV}$ and $1 \mathrm{keV}$ are almost identical, and that PSY increases linearly with the potential energy of the incident ion up to the highest charge state $(q=7)$ used in this study. These observations suggest that the DIET mechanism of the ion desorption confirmed by the ESD and PSD experiments for RGS can also be applied to the ion-induced potential sputtering process. This mechanism resembles the defect-mediated desorption model $[18,19]$ in the sense that an excited atom or an ion can initiate the desorption.

The $x$-axis intercept (i.e., the threshold energy of the potential sputtering) is found to be $\sim 40 \mathrm{eV}$ from the result of $E_{i}=500 \mathrm{eV}$. This energy is approximately twice the band-gap energy of solid Ne. The creation of two adjacent ions may lead to desorption of an ion due to Coulomb repulsion, although such a process has not been observed in photon-stimulated ion desorption experiments for solid Ar and solid $\mathrm{Kr}$ [5]. Another possibility is that a doubly excited atom is created in solid Ne, which desorbs from the surface, autoionizes, and is detected as $\mathrm{Ne}^{+}[20]$.

There is a difference between the two lines in Fig. 5, suggesting that the contribution of kinetic sputtering may not have been completely subtracted from the present results, even for $E_{i}=500 \mathrm{eV}$. The threshold energy obtained in the present study is the minimum value, and experiments with a lower incident ion energy are needed to determine the true threshold energy. 


\section{Acknowledgments}

The authors are grateful to Dr. Osamu Furuhashi for his significant contribution in the initial stages of this work. This work was partly supported by a Grant-in-Aid for Scientific Research from the Ministry of Education, Culture, Sports, Science and Technology, Japan, and by the Rikkyo University Special Fund for Research.

\section{References}

[1] Schwentner N, Koch E E and Jortner J 1985 Electronic excitations in condensed rare gases (Springer Tracts in Modern Physics vol 107) (Berlin: Springer-Verlag)

[2] Zimmerer G 1994 Nucl. Instrum. Meth. Phys. Res. B 91601

[3] Dujardin G, Philippe L, Rose M, Hirayama T, Ramage M J, Comtet G and Hellner L 1998 Appl. Phys. A 66527

[4] Hirayama T and Arakawa I 2006 J. Phys. Cond. Matt. 18 S1563

[5] Dujardin G, Hellner L, Besnard-Ramage M and Azria R 1990 Phys. Rev. Lett. 641289

[6] Hoshino A, Hirayama T and Arakawa I 1993 Appl. Surf. Sci. 70308

[7] Orth R G, Jonkman H T, Powell D H and Michl J 1981 J. Am. Chem. Soc. 103 60269

[8] David D E, Magnera T F, Tian R, Stulik D and Michl J 1986 Nucl. Instrum. Meth. Phys. Res. B 14378

[9] Balaji V, David D, Magnera T and Michl J 1990 Nucl. Instrum. Meth. Phys. Res. B 46435

[10] Hiraoka K, Watanabe M, Eguchi D, Okazaki S and Sato T 2002 Rapid Commun. Mass Spectrom. 161016

[11] Schou J, Ellegaard O, Sorensen H and Pedrys R 1988 Nucl. Instrum. Meth. Phys. Res. B 33808

[12] Ellegaard O, Schou J and Sørensen H 1986 Nucl. Instrum. Meth. Phys. Res. B 13567

[13] Pedrys R, Warczak B, Leskiewicz P, Schou J and Ellegaard O 1999 Nucl. Instrum. Meth. Phys. Res. B 157121

[14] Fujita S, Tachibana T, Koizumi T and Hirayama T 2009 J. Phys. Conf. Ser. 163 012083

[15] Märk T D and Scheier P 1987 Chem. Phys. Lett. 137245

[16] Haberland H 1985 Surf. Sci. 156305

[17] Tachibana T, Fukai K, Koizumi T and Hirayama T to be submitted

[18] Neidhart T, Pichler F, Aumayr F, Winter H, Schmid M and Varga P 1995 Phys. Rev. Lett. 745280

[19] Aumayr F, Varga P and Winter H 1999 Int. J. Mass Spectrom. 192415

[20] Wiethoff P, Ehrke H U, Menzel D and Feulner P 1995 Phys. Rev. Lett. 743792 